\title{
Abnormal development of sensory-motor, visual temporal and parahippocampal cortex in children with learning disabilities and borderline intellectual functioning
}

\author{
Francesca Baglio ${ }^{1}$, Monia Cabinio ${ }^{1,2}$, Cristian Ricci ${ }^{1,3}{ }^{\dagger}$, Gisella Baglio ${ }^{1}$, Susanna Lipari ${ }^{1}$, \\ Ludovica Griffanti ${ }^{1,4}$, Maria G. Preti ${ }^{1,4}$, Raffaello Nemni ${ }^{1,2}$, Mario Clerici ${ }^{1,2}$, Michela Zanette ${ }^{1}$ and \\ Valeria Blasi ${ }^{1}$ *
}

${ }^{1}$ IRCCS, Don Carlo Gnocchi Foundation - ONLUS, Milan, Italy

${ }^{2}$ Department of Pathophysiology and Transplantation, University of Milan, Milan, Italy

${ }^{3}$ Department of Epidemiology and Preventive Medicine, University of Regensburg, Germany

${ }^{4}$ Department of Electronics, Information and Bioengineering, Politecnico di Milano, Milan, Italy

\section{Edited by:}

Hans-Jochen Heinze, University of

Magdeburg, Germany

\section{Reviewed by:}

Zdravko Petanjek, University of

Zagreb, Croatia

Kerstin Krauel, University of

Magdeburg, Germany

\section{*Correspondence:}

Valeria Blasi, IRCCS, Don Carlo Gnocchi Foundation - ONLUS, Via Capecelatro 66, 20148 Milan, Italy e-mail: vblasi@dongnocchi.it

${ }^{\dagger}$ Present address:

Cristian Ricci, Department of Epidemiology and Preventive Medicine, University of Regensburg, Germany
Borderline intellectual functioning (BIF) is a condition characterized by an intelligence quotient (IQ) between 70 and 85 . BIF children present with cognitive, motor, social, and adaptive limitations that result in learning disabilities and are more likely to develop psychiatric disorders later in life. The aim of this study was to investigate brain morphometry and its relation to IO level in BIF children. Thirteen children with BIF and 14 age- and sexmatched typically developing (TD) children were enrolled. All children underwent a full IQ assessment (WISC-III scale) and a magnetic resonance (MR) examination including conventional sequences to assess brain structural abnormalities and high resolution 3D images for voxel-based morphometry analysis. To investigate to what extent the group influenced gray matter (GM) volumes, both univariate and multivariate generalized linear model analysis of variance were used, and the varimax factor analysis was used to explore variable correlations and clusters among subjects. Results showed that BIF children, compared to controls have increased regional GM volume in bilateral sensorimotor and right posterior temporal cortices and decreased GM volume in the right parahippocampal gyrus. GM volumes were highly correlated with $I Q$ indices. The present work is a case study of a group of BIF children showing that BIF is associated with abnormal cortical development in brain areas that have a pivotal role in motor, learning, and behavioral processes. Our findings, although allowing for little generalization to the general population, contribute to the very limited knowledge in this field. Future longitudinal MR studies will be useful in verifying whether cortical features can be modified over time even in association with rehabilitative intervention.

Keywords: borderline intellectual functioning (BIF), motor development, learning disabilities, brain maturation, voxel-based morphometry (VBM)

\section{INTRODUCTION}

In the last decades great attention has been devoted to specific learning disabilities leading to a significant improvement in diagnosis, treatment, and also detection of the neuroanatomical bases of these conditions. Among the school age children population, learning difficulties are very frequent, but the majority of these children do not meet the diagnostic criteria for specific learning disorders and fall into the non-specific learning disability category. In this group of children the great majority meet criteria for borderline intellectual functioning (BIF), which is a complex clinical entity that has received very little scientific investigation.

Borderline intellectual functioning has been described by the Diagnostic and Statistical Manual (DSM-IV-TR; American Psychiatric Association, 2000) to describe an intelligence quotient (IQ) range that is between 1 and $2 \mathrm{SD}$ below the mean (IQ: 70-85) and it is conceptualized as the frontier that delimits normal intellectual functioning from intellectual disability (American
Association of Mental Retardation [AAMR], 2002). According to recently published guidelines, BIF is a meta-condition characterized by heterogeneous cognitive difficulties, with a borderline IQ (between 71 and 85), and a deficit in personal functioning affecting daily and social activity (Salvador-Carulla et al., 2013). Its prevalence among school-aged population is estimated to be around 7\% (Karande et al., 2008). BIF is not a single neurodevelopmental syndrome and for this reason it is not possible to trace back to a single functioning profile (Salvador-Carulla et al., 2013). The heterogeneity in the clinical profile of BIF is also reflected in the many different factors contributing to this condition: genetic liability; biological causes, i.e., perinatal adverse events, and epigenetic factors such as socioeconomic status (SES) and maternal stress (Aicardi, 1998; Bradley and Corwyn, 2002; Marcus Jenkins et al., 2014). Nevertheless, the majority (about $60 \%$ ) of BIF children have a normal clinical profile regarding perinatal history, milestones, physical appearance, 
physical health, and middle class SES (Karande et al., 2008). BIF is a lifelong condition with obvious drawbacks in terms of social and vocational opportunities and health outcomes (Karande et al., 2008; Blacher et al., 2009; Emerson et al., 2010). Moreover, strong associations between borderline IQ and mental disorders in adult age (e.g., antisocial personality disorder, depression, suicide, and substance abuse) have been demonstrated (Douma et al., 2007; Ali and Hassiotis, 2008; Ferrari, 2009; Koenen et al., 2009; Emerson et al., 2010, 2011; Hassiotis et al., 2011).

Borderline intellectual functioning children typically present with intellectual (e.g., learning, reasoning, and problem solving) and adaptive skill difficulties (e.g., age-inappropriate social, communication, and daily living behaviors; Gresham and Elliott, 1987) and often show some degree of motor skill limitations (Frey and Chow, 2006; Fernell and Ek, 2010; Vuijk et al., 2010). Although BIF children have learning difficulties, these are not confined to a specific domain such as reading and writing (Karande et al., 2008). Moreover, BIF children often show some degree of written and spoken language comprehension deficits (SalvadorCarulla et al., 2013). With specific learning disorders they share poor performances in working memory (WM) and in short term memory (STM) tasks and consequent slowness in the execution of tasks requiring these abilities. Moreover, unlike specific learning disorders the adaptive skills limitation in social and academic areas of BIF children and most of all their learning difficulties reflect general intellectual functioning limitations with problems in attention, executive functions, gross, and fine motor abilities with lack of compensatory strategies (Cornoldi et al., 2014).

The advent of magnetic resonance imaging (MRI) allowed the quantification and visualization of structural brain changes in vivo, using specific morphometric techniques such as voxelbased morphometry (VBM; Ashburner and Friston, 2000), and FreeSurfer (Fischl et al., 1999), making feasible the investigation of brain structure also during development. In particular, the VBM technique is a widely used automatic technique that enables whole-brain analyses without a-priori hypotheses permitting the identification of structural differences between brains. A growing number of MRI studies investigated both normal (Giedd et al., 1999; Wilke and Holland, 2003; Sowell et al., 2004; Shaw et al., 2006) and abnormal (Sporn et al., 2003; Ridler et al., 2006; Paus et al., 2008) brain changes during development, showing that the majority of neuropsychiatric disorders are associated with deviations from normal brain development during childhood and/or adolescence. Despite the high prevalence and great social impact of BIF, there are no studies that investigated the brain characteristics in children with BIF using MRI.

The aim of this study was to determine if differences between BIF and typically developing (TD) children in terms of IQ level are also reflected in differences in gray matter (GM) brain volume and to identify factors able to explore for multiple correlations among these variables (IQ levels and GM volumes). To achieve this goal we collected brain MI structural images and IQ scores in 13 BIF children and 14 TD children. To limit the heterogeneity of the sample, we focused on BIF children with learning disabilities without the presence of genetic syndromes and/or major neuro-psychiatric disease, such as autism spectrum disorder or attentional deficit hyperactivity disorder (ADHD).

Due to the small number of subjects included in this study our study represents a case study of a group of BIF children with learning disabilities, allowing for little generalization of our findings to the general population of children with this condition, but contributing, for the first time, to the very limited knowledge in this field.

\section{MATERIALS AND METHODS PARTICIPANTS}

Twenty-three children with BIF referred to the Adolescence and Pediatric Neuropsychiatry Unit of our Institute in the year 2011 were enrolled. All BIF children were referred to our Unit from mainstream schools due to their learning difficulties.

Children were diagnosed as BIF according to the American Psychiatric Association's (2000). IQ scores, determined by means of the Wechsler Intelligence Scale for Children-III (WISC-III; Wechsler, 1991, 2006) ranged from 70 to 85 (mean Full Scale IQ score: FSIQ $80.26 \pm 4.15$ ). All BIF children underwent a clinical evaluation in order to exclude a neuropsychiatric disorder (such as ADHD and autism spectrum disorder), neurological conditions (epilepsy and traumatic brain injury), malformations or systemic diseases. All children included in the study had never taken medications, particularly referring to current or past psychostimulants, antidepressants, benzodiazepines and/or, antiepileptic drugs. None of the children included in the study were affected by genetic syndromes such as Down syndrome or Fragile X syndrome, nor had a positive history for systemic diseases, such as diabetes or immune disorders, nor infectious disease involving the central nervous system. Moreover, a detailed anamnesis that included pregnancy and perinatal history, developmental milestones, and associated disabilities (motor and language development and adaptive behavior) was collected for each BIF individual. All BIF children underwent a full learning abilities examination: reading and writing abilities were tested through a battery for developmental dyslexia and dysorthographia evaluation (DDE-2; Sartori and Job, 2007) and a reading test for primary school (Cornoldi and Colpo, 1998); visuo-spatial and motor integration skills were assessed using the developmental test of visual motor integration (VMI; Beery and Buktenica, 2000), while mathematic reasoning and calculation were tested with a battery for developmental dyscalculia (BDE; Biancardi and Nicoletti, 2004) or an assessment of math calculation and problem solving (AC-MT 6-11; Cornoldi et al., 2005). BIF children also underwent a full neuropsychological evaluation by means of: Neuropsychological Evaluation Battery for children (BVN 5-11; Bisiacchi et al., 2005); the Test of Reception of Grammar (TROG; Bishop, 2003); Modified Barrage bell test (Biancardi and Stoppa, 1997); Tower of London (TOL; Shallice, 1982; Sannio Fancello et al., 2006). Emotional and behavioral problems were assessed using the Child Behavioral Checklist (Achenbach and Rescorla, 2001, 2007).

The control group included fourteen TD children (M:F/7:7), with no history of neurological, psychiatric, or systemic disease or learning disability, selected from mainstream schools to be group matched to the BIF individuals for sex and age. 
All children underwent a full IQ assessment by means of the WISC-III scale within 2 weeks of the MRI session and assessment of the socioeconomic status (SES; by Hollingshead's (2011) Index of Social Position). The age range of recruited subject was 7-14 years for both groups. Table 1 summarizes demographic characteristics, as well as IQ scores and SES, while Table 2 shows the clinical data for each BIF child. The present study was approved by the scientific and ethics committees of our institution. All parents gave written informed consent for participation in the study.

\section{MRI PROTOCOL}

Magnetic resonance imaging was performed using a 1.5 Tesla MR system (Siemens Magnetom Avanto, Erlangen, Germany). The MRI protocol consisted of: (1) a conventional MRI study including T2-weighted $(\mathrm{TR} / \mathrm{TE}=2920 / 22 \mathrm{~ms}$; FoV $=240 \mathrm{~mm}$, slice thickness $=4 \mathrm{~mm}$, number of axial slices $=25)$ and fluid attenuated inversion recovery (FLAIR: TR/TE $=9000 / 121 \mathrm{~ms}$; FoV $=240 \mathrm{~mm}$, slice thickness $=5 \mathrm{~mm}$, number of coronal slices $=24)$ scans to assess anatomical structure and to detect the presence of brain abnormalities; (2) High-resolution 3D T1

Table 1 | Demographic characteristics of BIF and TD groups and neuropsychological evaluation of BIF.

\begin{tabular}{|c|c|c|c|}
\hline & BIF & TD & Group comparison \\
\hline \multicolumn{4}{|l|}{ Demographic } \\
\hline Age (in years) [median (IQR)] & $9.00[8.56-9.62]$ & $9.50[8.50-11.75]$ & $0.090^{\#}$ \\
\hline Sex $[M / F]$ & $8 / 5$ & $7 / 7$ & $0.963^{\S}$ \\
\hline SES [median $(95 \% \mathrm{CI})]$ & $3(1.53-3.47)$ & $4(3-4.10)$ & $0.008^{\#}$ \\
\hline FSIQ [median (IQR)] & 80 [77-83] & 118 [107-120] & $<0.0001^{\#}$ \\
\hline VIO [median (IQR)] & 84 [77-86] & 113.5 [103.25-118.25] & $<0.0001^{\#}$ \\
\hline PIQ [median (IOR)] & 82 [79-85] & 116 [105-118] & $<0.0001^{\#}$ \\
\hline \multicolumn{4}{|c|}{ Neuropsychological assessment } \\
\hline BVN 5-11 backward digit spa & & $-0,30[-2,12-0,70]$ & $3 / 13$ \\
\hline BVN 5-11 spatial span [medi & & $-0,75[-2,55-1,50]$ & $4 / 13$ \\
\hline \multicolumn{4}{|l|}{ Long term memory } \\
\hline BVN 5-11 word immediate re & (2R)] & $-0,59[-3,65-1,68]$ & $5 / 13$ \\
\hline BVN 5-11 word delayed reca & & $0,39[-4,45-1,76]$ & $2 / 13$ \\
\hline BVN 5-11 verbal paired asso & median (IQR)] & $-0,68[-2,15-0,58]$ & $6 / 13$ \\
\hline \multicolumn{4}{|l|}{ Language } \\
\hline BVN 5-11 naming [median (I & & $-1,00[-1,80-0,96]$ & $7 / 13$ \\
\hline TROG-2 [median (IQR)] & & $92,00[64-111]$ & $3 / 13$ \\
\hline \multicolumn{4}{|l|}{ Socio-emotional level } \\
\hline CBCL 6-18-Full Scale score [ & & 58 [34-69] & $6 / 13$ \\
\hline CBCL 6-18 internalizing [me & & 59 [33-69] & $6 / 13$ \\
\hline CBCL 6-18 externalizing [me & & 50 [32-59] & $0 / 13$ \\
\hline
\end{tabular}

IQR, Interquartile range; SES, socioeconomic status by Hollingshead Index of Social Position; WISC-III, Wechsler Intelligent Scale for Children-III (standard score); FSIQ, full scale IQ; VIQ, verbal IQ; PIQ, performance IQ; BVN 5-11, Neuropsychological Evaluation Battery for Children 5-11 years old (z-score); TROG-2, the Test of Reception Of Grammar-2 (standard score); TCM, modified barrage bells test (z-score); TOL, Tower of London (z-score); CBCL, Child Behavioral Check-List 6-18 (T-score). $N\left({ }^{*}\right)$, Number of subjects with borderline or pathological score. Data analyzed by chi-square (§) or Mann-Whitney U-test (\#). 
Table 2 | Demographic, anatomical MR, and clinical characteristics of BIF children.

\begin{tabular}{|c|c|c|c|c|c|}
\hline Subject, sex & $\begin{array}{l}\text { Conventional MR (brain } \\
\text { abnormalities) }\end{array}$ & $\begin{array}{l}\text { Pregnancy and } \\
\text { perinatal history }\end{array}$ & $\begin{array}{c}\text { Developmental } \\
\text { milestones [MD/LD] }\end{array}$ & $\begin{array}{c}\text { Comorbidity } \\
\text { [L/M/B] }\end{array}$ & SES \\
\hline $2, M$ & CC: Body, splenium & - & MD & $\mathrm{L} / \mathrm{M} / \mathrm{B}$ & 3 \\
\hline $3, F$ & - & - & MD & L/M/B & 2 \\
\hline $6, F$ & - & $\begin{array}{c}\text { CS (risk of } \\
\text { pre-eclampsia); NIC } \\
\text { (discharge at } 5 \mathrm{~d} \text { ) }\end{array}$ & - & M & 4 \\
\hline $8, M$ & - & $\begin{array}{c}\text { GE: 37; CS (abruptio } \\
\text { placentae) }\end{array}$ & LD & $L / B$ & 3 \\
\hline $9, F$ & - & - & - & L/B & 1 \\
\hline $10, F$ & - & - & - & L & 2 \\
\hline $11, M$ & - & - & LD & L & 3 \\
\hline $12, \mathrm{M}$ & - & - & - & L & 1 \\
\hline $13, F$ & - & - & - & L & 1 \\
\hline
\end{tabular}

Conventional MR: CC, thinning of corpus callosum; WMHs, white matter hyperintensities; VE, ventricular system enlargement; Clinical perinatal history: GE, gestational age at birth (weeks); CS, cesarean section; NIC, neonatal intensive care; Developmental milestones: MD, motor delay; LD, language delay. Comorbidity: $L$, language disabilities (expressive or mixed); $M$, motor disturbances; $B$, adaptive behavior disabilities; SES, socioeconomic status by Hollingshead Index of Social Position (1 lower, 2 lower-middle, 3 middle, 4 upper-middle, 5 Upper).

image $(\mathrm{TR} / \mathrm{TE}=1900 / 3.37 \mathrm{~ms}$, matrix size $=192 \times 256$, in-plane resolution $=1 \times 1 \mathrm{~mm}^{2}$, slice thickness $=1 \mathrm{~mm}$, number of axial slices $=176$ ) for VBM analysis.

Magnetic resonance anatomical and structural images obtained for all children were evaluated by an expert neuroradiologist blinded to the diagnosis.

Noteworthy, only 13 out of the 23 BIF children enrolled had MRI scan free from movement artifacts, while all 14 control children included in the study had good quality MRI scans.

All children were introduced to the MR protocol by a psychologist who trained them with play therapy and a mock scanner. During MRI all children had a video-goggle system to view movies.

\section{DATA ANALYSIS}

We compared the BIF and TD groups on demographic, IQ level using the chi-square test and Mann-Whitney $U$-test for categorical and dimensional variables, respectively.

\section{Voxel-based morphometry}

Voxel-based morphometry with diffeomorphic anatomical registration using exponentiated lie algebra (DARTEL; Ashburner and Friston, 2000; Ashburner, 2007) was conducted for the image analysis as described in Taki et al. (2012) on 13 BIF and all TD children
(10 BIF children were excluded due to motion artifacts). T1weighted 3D MR images were analyzed using Statistical Parametric Mapping $8^{1}$ in Matlab (Math Works, Natick, MA, USA). GM, white matter (WM), and cerebrospinal fluid (CSF) were extracted and normalized using a Bayesian approach (Unified Segmentation; Ashburner and Friston, 2005), then these segmented tissue maps were used to create a custom, population-specific template using the DARTEL template creation tool (Ashburner, 2007), and all images were finally normalized to the MNI space. After spatial processing, statistical analyses were performed using SPM8 and VBM8 software ${ }^{2}$. Total intracranial volume (TIV) was included in the second-level analysis as nuisance variable. For exploratory purposes, non-hypothesized group differences were only considered if a cluster of 50 or more contiguous voxels exceeded an uncorrected $p<0.001$ threshold at the local maximum voxel. We choose this permissive statistical threshold due to the small number of subjects included in this analysis (13 BIF and 14 TD children), and to the fact that no previous studies were available, limiting a priori hypotheses. The obtained clusters were then used to define the regions of interest (ROIs) for subsequent analyses.

\footnotetext{
${ }^{1}$ http://www.fil.ion.ucl.ac.uk/spm

${ }^{2}$ http://dbm.neuro.uni-jena.de/vbm/
} 


\section{Statistical analysis on ROI variables and IQ scores}

We computed the number of GM voxels falling within each ROI for each subject, after thresholding the GM maps (75\% of GM intensity value) by means of customized scripts. The obtained values represent numerical indices of GM volume for each subject, and were included in statistical analyses along with WISC-III indices (Table 3). Continuous variables were described using median and interquartile range according to variable skewness. Noncontinuous variables were described by percentages. To reduce skewness and to rescale variables to a common scale the normit Blom (Blom, 1958) transformation was used. Finally, missing data $(0.7 \%$ with sparse pattern between both control and analysis group) were imputed via the expectation maximization (EM) method (Dempster et al., 1977).

Both univariate and multivariate generalized linear model analysis of variance were used to investigate to what extent the group influenced GM volume. The multivariate test for the Hypothesis of No Overall Group Effect was reported using Wilks' Lambda test. Least Square means and 95\% confidence limits of normalized scores, $R$-square and $p$-values are reported in Table 4.

The oblique equamax factor analysis (Yaremko et al., 1986) of statistically significant ROI variables and IQ scores was used according to the hypothesis of a non-null correlation between factors to explore variable correlations and clusters among children. The Guttman-Kaiser criterion (Guttman, 1954) was used to determine the number of factors to retain after principal

Table 3 | Descriptive statistics of outcomes variables by group.

\begin{tabular}{|c|c|c|c|c|}
\hline \multirow[b]{2}{*}{ Variable } & \multicolumn{2}{|c|}{ Group TD $N=14$} & \multicolumn{2}{|c|}{ Group BIF $N=13$} \\
\hline & $\begin{array}{l}\text { Voxel } n \\
\text { Median }\end{array}$ & $\begin{array}{c}\text { Interquartile } \\
\text { range }\end{array}$ & $\begin{array}{l}\text { Voxel } n \\
\text { median }\end{array}$ & $\begin{array}{c}\text { Interquartile } \\
\text { range }\end{array}$ \\
\hline LPC-ROI1 & 211.5 & [205.25-217.5] & 221 & [209-237] \\
\hline LSM1-ROI2 & 76.5 & [66.5-100.75] & 148 & [126-156] \\
\hline RPTC-ROI3 & 85.5 & [64.25-95.75] & 127 & [117-150] \\
\hline RSM1-ROI4 & 17.5 & [13-27.5] & 40 & [30-50] \\
\hline RMOC-ROI5 & 130 & [97-162] & 127 & [110-146] \\
\hline LMOC-ROI6 & 58 & [49-60] & 50 & [42-60] \\
\hline RPH-ROI7 & 328 & [325-343] & 309 & [296-330] \\
\hline FSIO & 118 & [107-120] & 80 & [77-83] \\
\hline $\mathrm{PIO}$ & 116 & [105-118] & 82 & [79-85] \\
\hline VIO & 113.5 & [103.25-118.25] & 84 & [77-86] \\
\hline $\mathrm{VCl}$ & 117 & [102.75-119] & 85 & [76-87] \\
\hline $\mathrm{POI}$ & 117.5 & [107-122.5] & 85.5 & [84.5-92.25] \\
\hline FDI & 101.5 & [93.25-108.25] & 85 & [75-91] \\
\hline PSI & 103 & [95.5-106] & 74 & [70.25-77.5] \\
\hline
\end{tabular}

$R O I$, region of interest; voxel $n$ median, number of voxels included in the ROI median value; $L P C$, left posterior cingulated; $L S M 1$, left sensorimotor cortex; $R P T C$, right posterior temporal cortex; RSM1, right sensorimotor cortex; $R M O C$, right middle occipital cortex; $L M O C$, left middle occipital cortex; $R P H$, right parahippocampal gyrus; FSIQ, full scale IQ; PIQ, performance IQ; VIQ, verbal 1Q; VCl, verbal comprehension index; POI, perceptual organization index; FDI, freedom from distractibility index; PSI, processing speed index. component analysis (PCA). Biplots of retained factors, variances explained, and variables loadings on rotated factors are reported in Figure 1, groups are described by means of factor's centroids, $95 \%$ confidence limits of centroids are reported using ellipsoids of factor's scores. Sub-cluster within groups were obtained using the un-weighted pair-group centroid method and reported using a tree-plot (Figure 1).

All statistical evaluations were performed using the SAS software package version 9.2., statistical tests were two-tailed and the alpha value of 0.05 was considered.

\section{RESULTS}

\section{DEMOGRAPHIC CHARACTERISTICS, INTELLECTUAL FUNCTIONING,} AND CLINICAL HISTORY

Children with BIF were not significantly different from TD children on the matching variable of age and sex (Table 1). Significant differences between the two groups were found in SES status by means of Hollingshead Index of Social Position (Table 1). History of perinatal incidences was positive in five BIF children (two children were born preterm, one of them had also cesarean section (CS) for oligohydramnios, while three had cesarean section due to preeclampsia with intensive care unit recovery in one case, for umbilical cord around neck with first Apgar 3/8 in one case and for abruptio placentae in the last), while eight had a history of delay and/or abnormal development of language and/or motor abilities (Table 2).

The assessment of learning abilities showed that all children with BIF presented with deficits in one or more areas: seven children in reading, three in both reading and writing, and three in the calculation area alone. Specific learning disability diagnosis was excluded because of the low general cognitive profile of the children (see Table 1). Finally, all BIF children showed a range of comorbidities involving motor skills, language abilities, and adaptive behaviors. Perinatal and developmental history was negative in all TD children, with regular pregnancy and partum, and normal developmental milestones.

\section{MR ANATOMICAL DATA RESULTS}

Magnetic resonance anatomical and structural images obtained from 13 BIF and 14 TD revealed the presence of brain abnormalities in three BIF children. One child presented with a thinning of the splenium and the body of the corpus callosum, one had three non-specific small $(2-3 \mathrm{~mm})$ white matter hyperintensities (WMHs) in T2 and FLAIR images, and the last child presented with an enlargement of the ventricular system, not diagnosable as hydrocephalus. Given the nature of the brain alterations found in BIF children (minimal for dimension and involving selectively white matter) we decided to include them in the VBM analysis (that involved only GM, after segmentation of other brain tissues) after scrutiny of the accuracy of segmentation procedure. MR acquisition in TD children did not find any sign of brain abnormality.

\section{VBM RESULTS}

Voxel-based morphometry analysis showed differences in GM volumes in several areas (Figure 2; Table 4) in BIF compared to TD 
Table 4 | Univariate and multivariate analysis of variance on magnetic resonance data.

\begin{tabular}{|c|c|c|c|c|}
\hline MR variables $(*)$ & TD childrenLS-mean [95\% CL] & BIF childrenLS-mean $[95 \% \mathrm{CL}]$ & $R$-square & $p$-value \\
\hline LPC-ROI1 & $-0.27[-0.79 ; 0.024]$ & $0.29[-0.24 ; 0.83]$ & 0.0893 & 0.1300 \\
\hline RPTC-ROI3 & $-0.69[-1.05 ;-0.32]$ & $0.74[0.37 ; 1.12]$ & 0.5638 & $<0.0001$ \\
\hline RSM1-ROI4 & $-0.64[-1.03 ;-0.25]$ & $0.69[0.28 ; 1.09]$ & 0.4844 & $<0.0001$ \\
\hline $\mathrm{RPH}-\mathrm{ROI7}$ & $0.50[0.04 ; 0.95]$ & $-0.54[-1.01 ;-0.06]$ & 0.2938 & 0.0035 \\
\hline \multirow[t]{2}{*}{ MANOVA Results } & Wilk's $\lambda$ & F-statistic & Degrees of freedom & $p$-value \\
\hline & 0.173 & 12.94 & 7 & $<0.0001$ \\
\hline
\end{tabular}

(*) Blom's normit score of MR (magnetic resonance) variables; ROI, region of interest; LPC, left posterior cinguate; LSM1, left sensorimotor cortex; RPTC, right posterior temporal cortex; RSM1, right sensorimotor cortex; $R M O C$, right middle occipital cortex; $L M O C$, left middle occipital cortex; RPH, right parahippocampal gyrus.

children; these areas were included in the subsequent ROIs analysis as described in the methods' section. In particular, BIF versus TD showed: (1) Increased GM volume in left posterior cingulate (LPC-ROI 1, -11, -52, $6 \mathrm{x}, \mathrm{y}, \mathrm{z}$ MNI space; $k=357 ; z=4.24$ ), left sensorimotor cortex (LSM1-ROI2, -33, -30, 63 MNI space; $k=1306 ; z=3.89$ ), right posterior temporal cortex (RPTC-ROI3, $38,-72,10 \mathrm{x}, \mathrm{y}, \mathrm{z}$ MNI space; $k=246 ; z=3.74$ ) and right sensorimotor cortex (RSM1-ROI4, 33, -28, $61 \mathrm{x}, \mathrm{y}, \mathrm{z}$ MNI space; $k=327 ; z=3.64 ; 2)$ decreased GM in right middle occipital cortex (RMOC-ROI5, 29, -81, $15 \mathrm{x}, \mathrm{y}, \mathrm{z}$ MNI space; $k=239$;
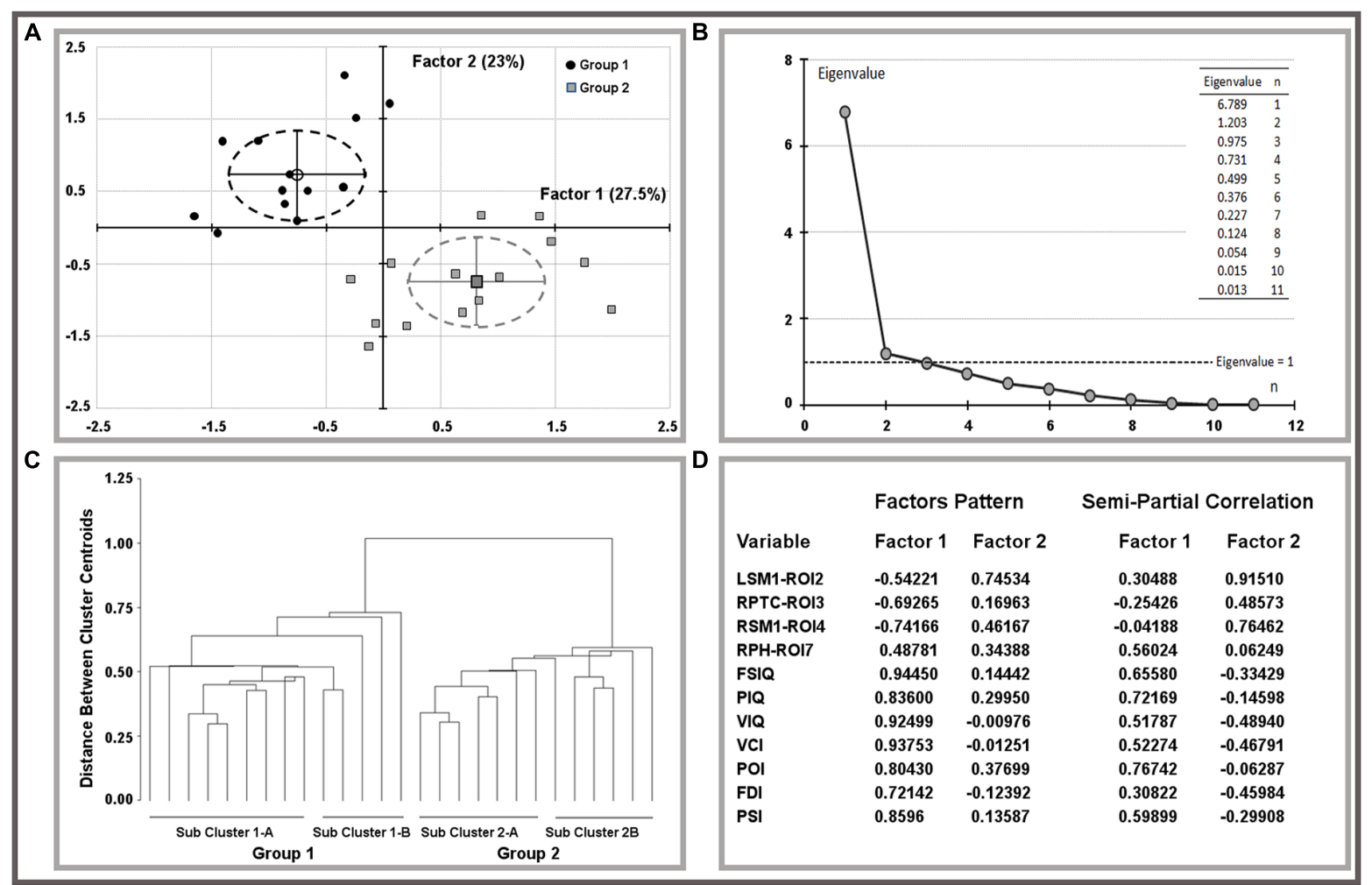

FIGURE 1 | Results of factor analysis on ROI variables and IQ scores. Biplots of factor scores by group (A) and related multivariate analysis (B,C). Explained variances and factor pattern were reported on (D). LSM1, left sensorimotor cortex; RPTC, right posterior temporal cortex; RSM1, right sensorimotor cortex; $\mathrm{RPH}$, right parahippocampus; FSIQ, full scale IQ; PIO, performance IQ; VIQ, verbal IQ; $\mathrm{VCI}$, verbal comprehension index; $\mathrm{POI}$, perceptual organization index; FDI, freedom from distractibility index; PSI, processing speed index. 


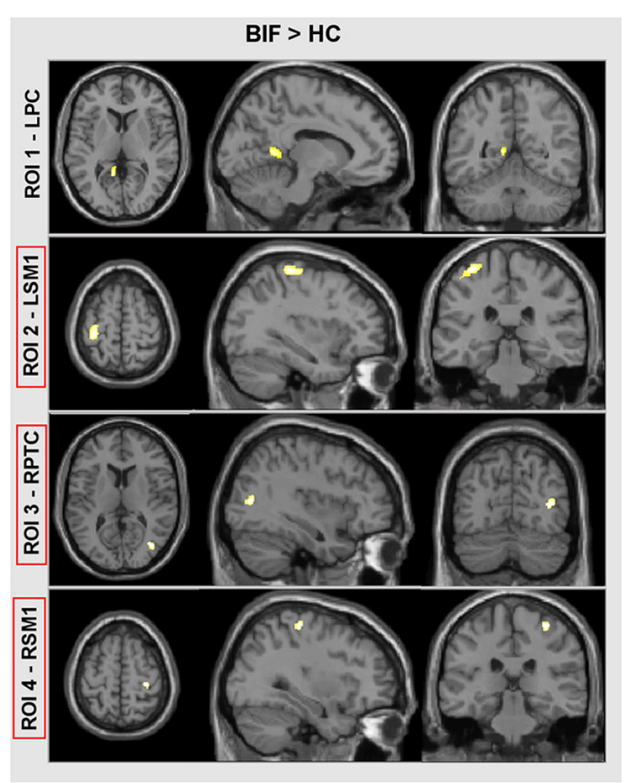

FIGURE 2 |VBM results. Direct comparison between BIF and TD. In red squares, cortical areas confirmed by the univariate test for group effect (see "Materials and Methods" for further details and statistical thresholds). ROI, region of interest; LPC, left posterior

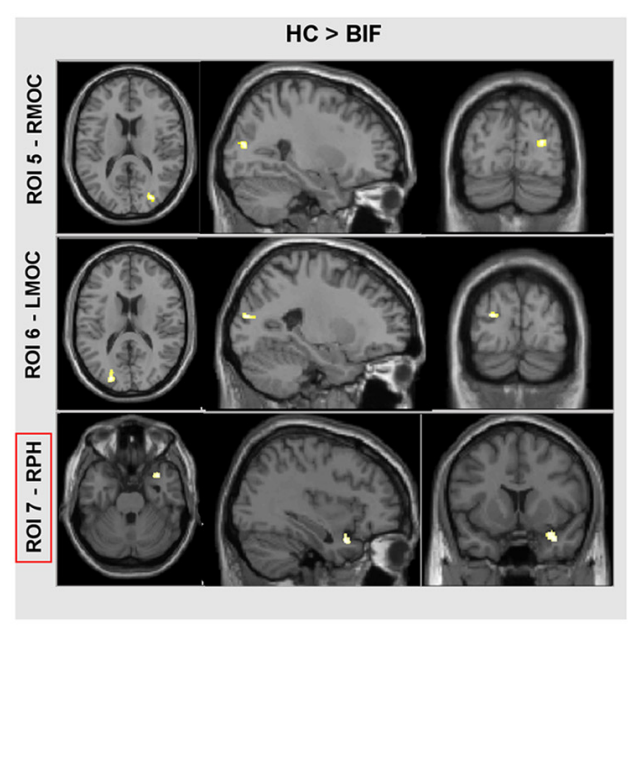

cingulate; LSM1, left sensorimotor cortex; RPTC, right posterior temporal cortex; RSM1, right sensorimotor cortex; RMOC, right middle occipital cortex; LMOC, left middle occipital cortex; and $\mathrm{RPH}$, right parahippocampus. $z=4.17$ ), left middle occipital cortex (LMOC-ROI6, $-24,-87$, $16 \mathrm{x}, \mathrm{y}, \mathrm{z}$ MNI space; $k=276 ; z=3.27)$, right parahippocampal gyrus (RPH-ROI 7, 36, 11, $-27 \mathrm{x}, \mathrm{y}, \mathrm{z}$ MNI space; $k=700$; $z=3.64)$.

\section{RESULTS OF STATISTICAL ANALYSIS ON ROI VARIABLES AND IO SCORES}

Both MRI (ROIs data) and IQ (IQ scores) data were rather skewed and highly inter-correlated, descriptions are reported in Table 3. The univariate test for group effect resulted statistically significant for LSM1-ROI2, RPTC-ROI3, RSM1-ROI4, and RPHROI7, but not for LPC-ROI1, RMOC-ROI5, and LMOC-ROI6 (Table 4). When considering all ROI variables in the MANOVA analysis, a statistically significant group effect was also found $(p<0.0001$, Wilk's $\lambda=0.173, F=12.94, \mathrm{DF}=7)$. Finally, according to Guttman-Kaiser criterion, two principal components were retained when both statistically significant ROI and IQ variables were considered in PCA (Figure 1). After oblique equamax factor analysis, ROI, and IQ variables were found to be well associated with the first two factors resulting in a cumulative explained variance of $50.5 \%$. These factors discriminated children according to group, no other sub-clusters were found when considering covariates. Within groups sub-clusters obtained by un-weighted pair-group centroid cluster analysis were not significantly associated with any of the first two rotated factors. Factor 1 was positively correlated with all IQ scores and was principally related to full scale IQ (FSIQ), verbal IQ (VIQ) and verbal comprehension index (VCI), having factor loadings of 0.94 , 0.93 , and 0.92, respectively. Moreover, Factor 1 was also found to be inversely related to LSM1-ROI2 (factor loading $=-0.54$ ),
RPTC-ROI3 (factor loading $=-0.69$ ), and RSM1-ROI4 (factor loading $=-0.74$ ), and positively related to RPH-ROI7 (factor loading $=0.49$ ), showing a close relationship between brain size in different regions and intelligence. According to these findings we can then deduct that the increment in GM volume in bilateral sensorimotor cortex (LSM1-ROI2; RSM1-ROI4) and in RPTC (ROI3) is associated with lower intelligence, while increased GM volume in RPH (ROI7) is associated with higher intelligence. Differently, Factor 2 was positively correlated with all ROI data and was principally related to LSM-ROI2 and RSM-ROI4, having factor loadings of 0.74 and 0.46 , respectively. Moreover, Factor 2 was also found to be positively correlated with FSIQ (factor loading $=0.14$ ), performance IQ (PIQ: factor loading $=0.30$ ), and POI (factor loading $=0.38$ ) and slightly negatively correlated with verbal subscales (VCI factor loading $=-0.013$ and VIQ factor loading $=-0.010$ ). This second factor illustrates how the differential components of the intelligence (verbal versus performance related functions) are influenced by brain maturation (Figure 1D).

\section{DISCUSSION}

Data herein demonstrate the presence of abnormal GM development in BIF children with learning disabilities that correlates with IQ levels.

The results of VBM analysis offer important additional data unraveling that BIF with learning disabilities is associated with abnormal cortical and subcortical GM development. Our data showed that BIF children, compared to controls have increased regional GM volume in bilateral sensorimotor and right posterior temporal cortices and decreased GM volume in the right 
parahippocampal gyrus. It is frequent observation that children with intellectual disabilities (ID) also show motor development disabilities and/or delays with a high correlation between motor and executive functions (Hartman et al., 2010), and between the degree of ID and performance of manual dexterity (Vuijk et al., 2010).

The close relationship between motor skills and IQ development was first argued by Piaget (1984), whose studies linked the development of thought with the emergence of skilled action. More recently, a study (Wassenberg et al., 2005) on a large cohort of TD children found a positive correlation between motor and cognitive abilities such as WM, verbal fluency, visuomotor abilities. Moreover, motor and intellectual skills share some neural substrates in the prefrontal cortex and the neocerebellum, which are strictly correlated in terms of phylogenetic development (Diamond, 2000) and of activation during motor and cognitive tasks (Raichle etal., 1994). A recent longitudinal study on teenagers (Ramsden etal., 2011) showed that changes over time in verbal IQ (VIQ) correlated with changes in the left motor cortex. Finally, a positive correlation between visuomotor abilities and IQ score, and between intelligence and GM volume in TD children, has recently been demonstrated (Pangelinan et al., 2011). These data are in line with our results showing that BIF children have increased GM volume in the posterior temporal cortex, a region that is part of the visual dorsal stream whose role is to mediate the visual control of skilled actions such as grasping (Milner and Goodale, 2008).

All these evidences highlight the importance of motor skills and visuomotor integration in the development of intelligence. Our study provides the first neuroanatomical demonstration of this link by showing that motor and visuomotor cortices are "abnormally" developed in children with BIF.

An additional result of our analyses concerns the parahippocampal gyrus, an area that showed an increment in size in the TD group. The parahippocampal gyrus and other areas of the medial temporal lobe have generally been implicated in episodic memory formation and learning (Paller and Wagner, 2002; Phelps, 2004) and learning processes in which the social and emotional context is crucial (Bhatt et al., 2012). Our results, showing decreased volume in the parahippocampal gyrus in our sample of BIF children, can shed some light on the neural mechanisms of learning difficulties and lack of social competencies typical of BIF children. Interestingly, a correlation between hippocampal volume and SES has been recently demonstrated Noble etal. (2012). This relation is probably due to the association of lower SES with higher exposure to stress (Buss et al., 2007; McEwen and Gianaros, 2010; Tottenham and Sheridan, 2010). SES is indeed a well-known factor associated with the BIF condition (Karande etal., 2008; Hassiotis et al., 2011) and also in our cohort this factor was significantly different between the two groups.

A novel and relevant aspect of these data is the integration of brain volumetric and clinical data related to the IQ. Results of the PCA statistical analysis revealed that two factors were able to discriminate the groups (TD versus BIF). The first factor was inversely related to the size of left and right sensorimotor cortices and posterior temporal cortex, positively related to the size of the parahippocampal gyrus and all IQ indices. This factor can be interpreted as related to the cortical and subcortical brain development. During childhood and adolescence the cortical and subcortical components of the brain change dramatically (Rosenzweig, 2003; Wilke et al., 2003; Lenroot and Giedd, 2006; Groeschel et al., 2010; Silk and Wood, 2011) with regionally specific, age-dependent variations (Wilke and Holland, 2003; Sowell et al., 2004). In fact, brain development is a dynamic process characterized by regressive, synapse elimination (pruning), and progressive changes, such as arborization, synaptogenesis, and an increase in pyramidal cell somata, influenced by genetical, epigenetical (environmental and experience-related) factors that determine cortical thickening or thinning (Cowan et al., 1984; Huttenlocher and Dabholkar, 1997; Rosenzweig, 2003; Juraska and Markham, 2004; Petanjek et al., 2008; Petanjek and Kostović, 2012). Sowell et al. (2004) demonstrated that, during childhood, the dorsal frontal region shows cortical thinning while perisylvian and temporal lobe GM structures, such as amygdala and hippocampus, increase in volume. According to these evidences we interpreted our data as due to delayed thinning mechanisms in sensorimotor and posterior temporal cortex, and delayed cortical thickening mechanisms in parahippocampal cortex. Such findings are highly relevant for the comprehension of the motor and cognitive difficulties of children with BIF and reveal that the size of these cerebral regions was a good predictor itself of the IQ allowing for the discrimination between groups.

The second factor correlates positively with FSIQ and performance IQ scores and negatively with VIQ scores. In the field of theoretical model of intelligence, there is a common distinction, strongly supported by neurobiological data (Blair, 2006; Horn and McArdle, 2007), between the crystallized and fluid intelligence. Factor 2 shows how differential components of intelligence are influenced by brain maturation. It seems that IQ scores related to the commonly defined "fluid intelligence" (performance scores) are the mostly correlated with brain volumes and in particular with the sensorimotor cortex.

Our sample of BIF children has different risk factors: biological and environmental: three had a positive conventional MRI structural scan and five had a positive perinatal history. Notably, the two aspects coincide only in one child. In this context, the above mentioned MR results are important as they represent the first attempt, to our knowledge, to detect brain abnormalities as a possible cause of BIF and underlie the necessity to investigate this aspect even in the absence of positive perinatal histories.

In our sample we found both biological and environmental evidences that may have influenced maternal stress during pregnancy and emotional interactions during infancy. As recently demonstrated, epigenetic factors can affect brain development in the intrauterine life (Raznahan et al., 2012) but also until the third decade of life (Petanjek et al., 2011). Moreover it has been demonstrate that BIF is a condition highly associated with mental health problems and socioeconomic disadvantage (Hassiotis et al., 1999; Emerson et al., 2010). According to the characteristics of our sample and to the recent scientific evidences linking 
cortical development and epigenetics (Petanjek and Kostović, 2012), we think that our study can represent a first evidence that BIF with learning disability is associated with abnormal cortical development and this is relevant for the timing of rehabilitative intervention.

In conclusion, data herein represent the first attempt to relate MRI structural markers with the clinical spectrum of the BIF condition in a pediatric sample. The importance of our results lie in the fact that even in a small group of subjects with heterogeneous characteristics, it is possible to detect abnormal cortical development. This implies that, whatever the cause of BIF, brain development is affected. This is very important as it underlies the importance of early detection of this condition.

The small number of subjects and the clinical heterogeneity of the BIF sample constitute a limitation of our study, this should be considered a case study of a group of BIF. Our data, though, are quite clear and robust in showing a cortical development delay and a significant correlation between cortical development and IQ scores. For this reason we think our data demonstrate that, whatever the clinical condition, learning disabilities with BIF are associated with brain development abnormalities. Future longitudinal MRI studies will be useful in verifying whether these characteristics are modifiable over time and if rehabilitative interventions could drive the brain maturation toward patterns that resemble those seen in TD.

\section{REFERENCES}

Achenbach, T. M., and Rescorla, L. A. (2001). Manual for the ASEBA School-Age Forms \& Profiles. Burlington, VT: University of Vermont, Research Center for Children, Youth, and Families.

Achenbach, T. M., and Rescorla, L. A. (2007). Multicultural Supplement to the Manual for the ASEBA School-Age Forms \& Profiles. Burlington, VT: University of Vermont Research Center for Children, Youth, and Families.

Aicardi, J. (1998). The etiology of developmental delay. Semin. Pediatr. Neurol. 5, 15-20. doi: 10.1016/S1071-9091(98)80013-2

Ali, A., and Hassiotis, A. (2008). Illness in people with intellectual disabilities. BMJ 336, 570-571. doi: 10.1136/bmj.39490.543137.80

American Association of Mental Retardation [AAMR]. (2002). Mental Retardation: Definition, Classification and System of Support, 10th Edn. Washington, DC: American Association on Intellectual and Developmental Disabilities.

American Psychiatric Association. (2000). Diagnostic and Statistical Manual of Mental Disorders, 4th Edn. Washington, DC: American Psychiatric Association.

Ashburner, J. (2007). A fast diffeomorphic image registration algorithm. Neuroimage 38, 95-113. doi: 10.1016/j.neuroimage.2007.07.007

Ashburner, J., and Friston, K. J. (2000). Voxel-based morphometry-the methods. Neuroimage 11, 805-821. doi: 10.1006/nimg.2000.0582

Ashburner, J., and Friston, K. J. (2005). Unified segmentation. Neuroimage 26, 839-851. doi: 10.1016/j.neuroimage.2005.02.018

Beery, K. E., and Buktenica, N. A. (2000). VMI, Developmental Test of Visual-Motor Integration. Il Beery-Buktenica con $i$ test Supplementari di Percezione Visiva e Coordinazione Motoria, Manuale, Traduzione e studio Italiano, a cura di Preda C (Firenze: O. S. Organizzazioni Speciali).

Bhatt, M. A., Lohrenz, T., Camerer, C. F., and Montague, P. R. (2012). Distinct contributions of the amygdala and parahippocampal gyrus to suspicion in a repeated bargaining game. Proc. Natl. Acad. Sci. U.S.A. 109, 8728-8733. doi: $10.1073 /$ pnas. 1200738109

Biancardi, A., and Nicoletti, C. (2004). Batteria per la Discalculia Evolutiva (BDE). Torino: Edizioni Omega.
Biancardi, A., and Stoppa, E. (1997). Il test delle campanelle modificato: una proposta per lo studio dell'attenzione in età evolutiva. Psichiatr. Infanzia Adolesc. 64, 73-84.

Bishop, D. V. M. (2003). The Test for Reception of Grammar, Version 2 (TROG-2). London: Pearson Assessment. [Italian version: Suraniti and Ferri e Neri, (2009). Firenze: Giunti Organizzazioni Speciali].

Bisiacchi, P. S., Cedron, M., Gugliotta, M., Tressoldi, P. E., and Vio, C. (2005). Batteria di Valutazione Neuropsicologica per l'età Evolutiva (BVN 5-11). Trento: Edizioni Erickson.

Blacher, J., Baker, B. L., and Eisenhower, A. S. (2009). Student-teacher relationship stability across early school years for children with intellectual disability or typical development. Am. J. Intellect. Dev. Disabil. 114, 322-339. doi: 10.1352/1944-7558114.5.322

Blair, C. (2006). How similar are fluid cognition and general intelligence? A developmental neuroscience perspective on fluid cognition as an aspect of human cognitive ability. Behav. Brain Sci. 29, 109-125. doi: 10.1017/S0140525X06009034

Blom, G. (1958). Statistical Estimates and Transformed Beta-Variables. New York: Wiley.

Bradley, R. H., and Corwyn, R. F. (2002). Socioeconomic status and child development. Annu. Rev. Psychol. 53, 371-399. doi: 10.1146/annurev.psych.53. 100901.135233

Buss, C., Lord, C., Wadiwalla, M., Hellhammer, D. H., Lupien, S. J., Meaney, M. J., et al. (2007). Maternal care modulates the relationship between prenatal risk and hippocampal volume in women but not in men. J. Neurosci. 27, 2592-2595. doi: 10.1523/JNEUROSCI.3252-06.2007

Cornoldi, C., and Colpo, G. (1998). Prove di Lettura MT per la Scuola Elementare-2, Manuale. Firenze: O. S. Organizzazioni Speciali.

Cornoldi, C., Giofrè, D., Orsini, A., and Pezzuti, L. (2014). Differences in the intellectual profile of children with intellectual vs. learning disability. Res. Dev. Disabil. 35, 2224-2230. doi: 10.1016/j.ridd.2014.05.013

Cornoldi, C., Lucangeli, D., and Bellina, M. (2005). Test AC-MC 6-11. Test di Valutazione delle Abilità di Calcolo. Trento: Edizioni Erickson.

Cowan, W. M., Fawcett, J. W., O'Leary, D. D., and Stanfield, B. B. (1984). Regressive events in neurogenesis. Science 225, 1258-1265. doi: 10.1126/science. 6474175

Dempster, A. P., Laird, N. M., and Rubin, D. B. (1977). Maximum likelihood from incomplete data via the EM algorithm. J. R. Stat. Soc. Ser. B 39, 1-38.

Diamond, A. (2000). Close interrelation of motor development and cognitive development and of the cerebellum and prefrontal cortex. Child Dev. 71, 44-56. doi: 10.1111/1467-8624.00117

Douma, J. C., Dekker, M. C., de Ruiter, K. P., Tick., N. T., and Koot, H. M. (2007). Antisocial and delinquent behaviors in youths with mild or borderline disabilities. Am. J. Ment. Retard. 112, 207-220. doi: 10.1352/08958017(2007)112[207:AADBIY]2.0.CO;2

Emerson, E., Einfeld, S., and Stancliffe, R. J. (2010). The mental health of young children with intellectual disabilities or borderline intellectual functioning. Soc. Psychiatry Psychiatr. Epidemiol. 45, 579-587. doi: 10.1007/s00127-0090100-y

Emerson, E., Einfeld, S., and Stancliffe, R. J. (2011). Predictors of the persistence of conduct difficulties in children with cognitive delay. J. Child Psychol. Psychiatr. 52, 1184-1194. doi: 10.1111/j.1469-7610.2011.02413.x

Fernell, E., and Ek, U. (2010). Borderline intellectual functioning in children and adolescents - insufficiently recognized difficulties. Acta Paediatr. 99, 748-753. doi: 10.1111/j.1651-2227.2010.01707.x

Ferrari, M. (2009). Borderline intellectual functioning and the intellectual disability construct. Intellect. Dev. Disabil. 47, 386-389. doi: 10.1352/1934-9556-47.5.386

Fischl, B., Sereno, M. I., and Dale, A. M. (1999). Cortical surface-based analysis. II: inflation, flattening, and a surface-based coordinate system. Neuroimage 9, 195-207 doi: 10.1006/nimg.1998.0396

Frey, G. C., and Chow, B. (2006). Relationship between BMI, physical fitness, and motor skills in youth with mild intellectual disabilities. Int. J. Obes. 30, 861-867. doi: 10.1038/sj.ijo.0803196

Giedd, J. N., Blumenthal, J., Jeffries, N. O., Castellanos, F. X., Liu, H., Zijdenbos, A., et al. (1999). Brain development during childhood and adolescence: a longitudinal MRI study. Nat. Neurosci. 2, 861-863. doi: 10.1038/13158

Gresham, F. M., and Elliott, S. N. (1987). The relationship between adaptive behavior and social skills issues in definition and assessment. J. Spec. Educ. 21, 167-181. doi: $10.1177 / 002246698702100115$ 
Groeschel, S., Vollmer, B., King, M. D., and Connelly, A. (2010). Developmental changes in cerebral grey and white matter volume from infancy to adulthood. Int. J. Dev. Neurosci. 28, 481-489. doi: 10.1016/j.ijdevneu.2010.06.004

Guttman, L. (1954). Some necessary conditions for common-factor analysis. Psychometrika 19, 149-161. doi: 10.1007/BF02289162

Hartman, E., Houwen, S., Scherder, E., and Visscher, C. (2010). On the relationship between motor performance and executive functioning in children with intellectual disabilities. J. Intellect. Disabil. Res. 54, 468-477. doi: 10.1111/j.1365-2788.2010.01284.x

Hassiotis, A., Tanzarella, M., Bebbington, P., and Cooper, C. (2011). Prevalence and predictors of suicidal behaviour in a sample of adults with estimated borderline intellectual functioning: results from a population survey. J. Affect. Disord. 129, 380-384. doi: 10.1016/j.jad.2010.10.002

Hassiotis, A., Ukoumunne, O., Tyrer, P., Piachaud J., Gilvarry C., Harvey K. et al. (1999). Prevalence and characteristics of patients with severe mental illness and borderline intellectual functioning. Report from the UK700 randomised controlled trial of case management. Br. J. Psychiatry 75, 135-140. doi: 10.1192/bjp.175.2.135

Hollingshead, A. B. (2011). Four factor index of social status. Yale J. Sociol. 8, 21-53

Horn, J., and McArdle, J. (2007). "Understanding human intelligence since spearman," in Factor Analysis at 100 Years, eds R. Cudeck and R. MacCallum (Mahwah, NJ: Erlbaum), 205-247.

Huttenlocher, P. R., and Dabholkar, A. S. (1997). Regional differences in synaptogenesis in human cerebral cortex. J. Comp. Neurol. 387, 167-178. doi: 10.1002/(SICI)1096-9861(19971020)387:2<167::AID-CNE1>3.0.CO;2-Z

Juraska, J. M., and Markham, J. A. (2004). The cellular basis for volume changes in the rat cortex during puberty: white and gray matter. Ann. N. Y. Acad. Sci. 1021, 431-435. doi: 10.1196/annals.1308.058

Karande, S., Kanchan, S., and Kulkarni, M. (2008). Clinical and psychoeducational profile of children with borderline intellectual functioning. Indian J. Pediatr. 75 795-800. doi: 10.1007/s12098-008-0101-y

Koenen, K. C., Moffitt, T. E., Roberts, A. L., Martin, L. T., Kubzansky, L., Harrington, H., et al. (2009). Childhood IQ and adult mental disorders: a test of the cognitive reserve hypothesis. Am. J. Psychiatry 166, 50-57. doi: 10.1176/appi.ajp.2008.08030343

Lenroot, R. K., and Giedd, J. N. (2006). Brain development in children and adolescents: insights from anatomical magnetic resonance imaging. Neurosci. Biobehav Rev. 30, 718-729. doi: 10.1016/j.neubiorev.2006.06.001

Marcus Jenkins, J. V., Woolley, D. P., Hooper, S. R., and De Bellis, M. D. (2014). Direct and indirect effects of brain volume, socioeconomic status and family stress on child IQ. J. Child Adolesc. Behav. 1, 1000107.

McEwen, B. S., and Gianaros, P. J. (2010). Central role of the brain in stress and adaptation: links to socioeconomic status, health, and disease. Ann. N. Y. Acad. Sci. 1186, 190-222. doi: 10.1111/j.1749-6632.2009.05331.x

Milner, A. D., and Goodale, M. A. (2008). Two visual systems re-viewed. Neuropsychologia 46, 774-785. doi: 10.1016/j.neuropsychologia.2007.10.005

Noble, K. G., Houston, S. M., Kan, E., and Sowell, E. R. (2012). Neural correlates of socioeconomic status in the developing human brain. Dev. Sci. 15, 516-527. doi 10.1111/j.1467-7687.2012.01147.x

Paller, K. A., and Wagner, A. D. (2002). Observing the transformation of experience into memory. Trends Cogn. Sci. 6, 93-102. doi: 10.1016/S1364-6613(00)01845-3

Pangelinan, M. M., Zhang, G., VanMeter, J. W., Clark, J. E., Hatfield, B. D., and Haufler, A. J. (2011). Beyond age and gender: relationships between cortical and subcortical brain volume and cognitive-motor abilities in schoolage children. Neuroimage 54, 3093-3100. doi: 10.1016/j.neuroimage.2010. 11.021

Paus, T., Toro, R., Leonard, G., Lerner, J. V., Lerner, R. M., Perron, M., et al. (2008). Morphological properties of the action-observation cortical network in adolescents with low and high resistance to peer influence. Soc. Neurosci. 3, 303-316. doi: 10.1080/17470910701563558

Petanjek, Z., Judas, M., Kostović, I., and Uylings, H. B. (2008). Lifespan alterations of basal dendritic trees of pyramidal neurons in the human prefrontal cortex: a layer-specific pattern. Cereb. Cortex 18, 915-929. doi: 10.1093/cercor/ bhm124

Petanjek, Z., Judaš, M., Šimić, G., Rašin, M. R., Uylings, H. B. M., Rakic, P., et al. (2011). Extraordinary neoteny of synaptic spines in the human prefrontal cortex. Proc. Natl. Acad. Sci. U.S.A. 108, 13281-13286. doi: 10.1073/pnas. 1105108108
Petanjek, Z., and Kostović, I. (2012). Epigenetic regulation of fetal brain development and neurocognitive outcome. Proc. Natl. Acad. Sci. U.S.A. 109, 11062-11063. doi: 10.1073/pnas.1208085109

Phelps, E. A. (2004). Human emotion and memory: interactions of the amygdala and hippocampal complex. Curr. Opin. Neurobiol. 14, 198-202. doi: 10.1016/j.conb.2004.03.015

Piaget, J. (1984). "Piaget's theory," in Handbook of Child Psychology, ed. P. Mussen (New York: John Wiley), 103-128.

Raichle, M. E., Fiez, J. A., Videen, T. O., MacLeod, A.-M. K., Pardo, J. V., Fox, P. T., et al. (1994). Practice-related changes in human brain functional anatomy during nonmotor learning. Cereb. Cortex 4, 8-26. doi: 10.1093/cercor/4.1.8

Ramsden, S., Richardson, F. M., Josse, G., Thomas, M. S. C., Ellis, C., Shakeshaft, C., et al. (2011). Verbal and non-verbal intelligence changes in the teenage brain. Nature 479, 113-116. doi: 10.1038/nature10514

Raznahan, A., Greenstein, D., Raitano Lee, N., Clasen, L. S., and Giedd, J. N. (2012). Prenatal growth in humans and postnatal brain maturation into late adolescence. Proc. Natl. Acad. Sci. U.S.A. 109, 11366-11371. doi: 10.1073/pnas.1203350109

Ridler, K., Veijola, J. M., Tanskanen, P., Miettunen, J., Chitnis, X., Suckling, J., et al. (2006). Fronto-cerebellar systems are associated with infant motor and adult executive functions in healthy adults but not in schizophrenia. Proc. Natl. Acad. Sci. U.S.A. 103, 15651-15656. doi: 10.1073/pnas.0602639103

Rosenzweig, M. R. (2003). Effects of differential experience on the brain and behavior. Dev. Neuropsychol. 24, 523-540. doi: 10.1080/87565641.2003.9651909

Salvador-Carulla, L., García-Gutiérrez, J. C., Ruiz Gutiérrez-Colosía, M., ArtigasPallarès, J., García Ibáñez, J., González Pérez, J., etal. (2013). Borderline intellectual functioning: consensus and good practice guidelines. Rev. Psiquiatr. y salud Ment. 6, 109-120. doi: 10.1016/j.rpsm.2012.12.001

Sannio Fancello, G., Vio, C., and Cianchetti, C. (2006). Test TOL - Torre di Londra. Trento: Edizioni Erickson.

Sartori, G., and Job, R. (2007). Batteria per la Valutazione della Dislessia e della Disortografia Evolutiva-2 (DDE-2). Firenze: O. S. Organizzazioni Speciali.

Shallice, T. (1982). Specific impairment of planning. Philos. Trans. R. Soc. Lond. B Biol. Sci. 298, 199-209. doi: 10.1098/rstb.1982.0082

Shaw, P., Greenstein, D., Lerch, J., Clasen, L., Lenroot, R., Gogtay, N., et al. (2006). Intellectual ability and cortical development in children and adolescents. Nature 440, 676-679. doi: 10.1038/nature04513

Silk, T. J., and Wood, A. G. (2011). Lessons about neurodevelopment from anatomical magnetic resonance imaging. J. Dev. Behav. Pediatr. 32, 158-168. doi: 10.1097/DBP.0b013e318206d58f

Sowell, E. R., Thompson, P. M., Leonard, C. M., Welcome, S. E., Kan, E., and Toga, A. W. (2004). Longitudinal mapping of cortical thickness and brain growth in normal children. J. Neurosci. 24, 8223-8231. doi: 10.1523/JNEUROSCI.179804.2004

Sporn, A. L., Greenstein, D. K., Gogtay, N., Jeffries, N. O., Lenane, M., Gochman, P., et al. (2003). Progressive brain volume loss during adolescence in childhood-onset schizophrenia. Am. J. Psychiatry 160, 2181-2189. doi: 10.1176/appi.ajp.160.12.2181

Taki, Y., Hashizume, H., Sassa, Y., Takeuchi, H., Asano, M., Asano, K., etal. (2012). Correlation among body height, intelligence, and brain gray matter volume in healthy children. Neuroimage 59, 1023-1027. doi: 10.1016/j.neuroimage.2011.08.092

Tottenham, N., and Sheridan, M. A. (2010). A review of adversity, the amygdala and the hippocampus: a consideration of developmental timing. Front. Hum. Neurosci. 3:68. doi: 10.3389/neuro.09.068.2009

Vuijk, P. J., Hartman, E., Scherder, E., and Visscher, C. (2010). Motor performance of children with mild intellectual disability and borderline intellectual functioning. J. Intellect. Disabil. Res. 54, 955-965. doi: 10.1111/j.1365-2788.2010. 01318.x

Wassenberg, R., Feron, F. J. M., Kessels, A. G. H., Hendriksen, J. G. M., Kalff, A. C., Kroes, M., et al. (2005). Relation between cognitive and motor performance in 5to 6-year-old children: results from a large-scale cross-sectional study. Child Dev. 76, 1092-1103. doi: 10.1111/j.1467-8624.2005.00899.x

Wechsler, D. (1991). WISC-III: Wechsler Intelligence Scale for Children. New York: The Psychological Corporation.

Wechsler, D. (2006). WISC-III. Contributo alla Taratura Italiana. Firenze: Giunti OS Wilke, M., and Holland, S. K. (2003). Variability of gray and white matter during normal development: a voxel-based MRI analysis. Neuroreport 14, 1887-1890. doi: 10.1097/01.wnr.0000090951.15465.c8 
Wilke, M., Sohn, J.-H., Byars, A. W., and Holland, S. K. (2003). Bright spots: correlations of gray matter volume with IQ in a normal pediatric population. Neuroimage 20, 202-215. doi: 10.1016/S1053-8119(03)00199-X

Yaremko, R. M., Harari, H., Harrison, R. C., and Lynn, E. (1986). Handbook of Research and Quantitiative Methods in Psychology. London: Lawrence Erlbaum Hillsdale.

Conflict of Interest Statement: The authors declare that the research was conducted in the absence of any commercial or financial relationships that could be construed as a potential conflict of interest.

Received: 21 January 2014; accepted: 22 September 2014; published online: 15 October 2014.
Citation: Baglio F, Cabinio M, Ricci C, Baglio G, Lipari S, Griffanti L, Preti MG, Nemni R, Clerici M, Zanette $M$ and Blasi V (2014) Abnormal development of sensory-motor, visual temporal and parahippocampal cortex in children with learning disabilities and borderline intellectual functioning. Front. Hum. Neurosci. 8:806. doi: 10.3389/fnhum.2014.00806

This article was submitted to the journal Frontiers in Human Neuroscience Copyright (C) 2014 Baglio, Cabinio, Ricci, Baglio, Lipari, Griffanti, Preti, Nemni, Clerici, Zanette and Blasi. This is an open-access article distributed under the terms of the Creative Commons Attribution License (CC BY). The use, distribution or reproduction in other forums is permitted, provided the original author(s) or licensor are credited and that the original publication in this journal is cited, in accordance with accepted academic practice. No use, distribution or reproduction is permitted which does not comply with these terms. 